\title{
Testing Causal Explanations of Viewing Time Measures of Sexual Interests
}

\author{
Alexander F. Schmidt \\ Johannes Gutenberg-University Mainz \\ Judith Koppehele-Gossel \\ VITOS Clinics Hochtaunus \\ Rainer Banse \\ University of Bonn \\ Roland Imhoff \\ Johannes Gutenberg-University Mainz
}

\begin{abstract}
Author Note
Alexander F. Schmidt, Department of Psychology, Social \& Legal Psychology, Johannes Gutenberg-University Mainz, Mainz, Germany; Judith Koppehele-Gossel, VITOS Clinics Hochtaunus, Friedrichsdorf-Köppern, Germany; Rainer Banse, Department of Psychology, Social \& Legal Psychology, University of Bonn, Bonn, Germany; Roland Imhoff, Department of Psychology, Social \& Legal Psychology, Johannes Gutenberg-University Mainz, Mainz, Germany.
\end{abstract}

Correspondence concerning this article should be addressed to Alexander F. Schmidt, Social \& Legal Psychology, Johannes Gutenberg-University Mainz, Binger Str. 14-16, D-55122 Mainz, Germany, alexander.schmidt@uni-mainz.de 


\begin{abstract}
The term viewing time (VT) effect refers to a phenomenon whereby respondents typically take longer to judge the sexual attractiveness of targets from sexually preferred (versus nonpreferred) categories. Although frequently characterized as an unobtrusive measure of respondents' sexually motivated reactions to the stimulus images themselves, the typical pattern of response times might be sufficiently explained by the task demands of the seemingly less relevant rating task. Utilizing three different VT variants, the present paper reports an experimental investigation $(N=136$ heterosexual women and men) that tested hypotheses derived from hot stimulus-based processes versus cold cognitive task-based processes. Specifically, stimulus-based processes would predict VT effects even without a rating task, greater VT effects for sexually more suggestive images, and correlations of VT effects with individual sex drive differences. The task-based processes would not imply such predictions, but instead suggest identical response patterns for abstract non-pictorial stimuli that require the same feature integration. Results unanimously speak to the relevance of task demands but provide no support for stimulus-based processes. Implications of these findings for the causal explanation of VT effects are discussed.
\end{abstract}

Key words: viewing time, indirect measure, sexual interest, sexual orientation, sex drive 
Testing Causal Explanations of Viewing Time Measures of Sexual Interests

\section{Introduction}

Over the last 25 years, numerous studies have shown that individuals' unobtrusively measured viewing times are prolonged for sexually preferred (versus non-preferred) stimuli when they are instructed to rate pictures of persons in terms of their subjectively perceived sexual attractiveness (e.g., Banse et al., 2010; Dawson et al., 2012; Ebsworth \& Lalumière, 2012; Harris et al., 1996; Israel \& Strassberg, 2009; Jahnke et al., 2021; Larue et al., 2014; Lippa, 2017; Oberlader et al., 2017; Petterson et al., 2018; Rönspies et al., 2015; Quinsey et al., 1996; $\mathrm{Xu}$ et al., 2017). This basic finding-in the literature commonly referred to as viewing time (VT) effect-is so robust, that it is frequently used as an easy-to-apply latency-based indirect measure of individual differences in sexual interests, particularly, in forensic contexts where self-report measures are not the most viable option due to positive self-presentation concerns (Schmidt \& Banse, in press; for systematic overviews see Pedneault et al., 2021; Schmidt et al., 2017).

For a long time, the basic VT effect had been accepted and exploited as a measurement tool without further scrutiny of the mediating psychological processes. But why exactly would respondents take longer for exemplars of their sexually preferred target categories? The standard responses were comparatively vague references to attentional processes such as an initial aesthetic response presumed to precede more complex physiological and behavioral responses in sexual arousal (e.g., Bourke \& Gormley, 2012; Gray et al., 2015; Kalmus \& Beech, 2005). Imhoff and colleagues (2010) first addressed this apparent lack of a more systematic understanding of the underlying architecture of VT effects in a series of four experimental studies. It was shown that VT effects remained diagnostic of participants' sexual orientation even under restricted task conditions: Presenting target pictures for considerably shortened stimulus intervals $(750 \mathrm{~ms}$ and $500 \mathrm{~ms})$ while the sexual attractiveness rating scale was displayed only after 
the targets on a separate page (with or without stimulus backward masking in between; Imhoff et al., 2010, Studies 1 and 2) ruled out attentional adhesion as an explanatory mechanism. Moreover, deliberate delay due to aesthetic indulging was ruled out as the restriction of the response time using a speeded binary-decision VT task with a 1,000ms response window did not impair task validity (Imhoff et al., 2010, Study 3) and utilizing heads-only stimuli also did not affect the diagnostic effect (Imhoff et al., 2010, Study 4). Based on their findings that VT effects emerged even under these carefully restricted task conditions, Imhoff and colleagues (2010) concluded two possible remaining explanations, one of them a (cold) cognitive byproduct of the task, the other one a (hot) process triggered by (even short) confrontation with a sexually attractive stimulus.

\section{Cold Cognitive Task Demands (Feature-Checking Hypothesis)}

The cold cognitive task demand explanation is based on the fact that most of the VT literature does not just record a passive "viewing time", but asks participants to judge the attractiveness of the presented stimuli. Hence, participants' decision times are measured. Typically seen as a way to conceal that researchers are actually interested in response times rather than self-reported responses, this inclusion of the rating task has substantial consequences. As argued by Imhoff and colleagues (2010), reaching a negative response ("sexually not attractive") requires processing less bits of information than a positive response ("sexually attractive"). A person who is sexually interested in handsome adult men can give a negative response as soon as it becomes apparent that the presented stimulus is either not an adult, nor male, nor handsome. One bit of mis-fitting information is thus sufficient to give a negative response. There is, however, an asymmetrically greater need for information integration in order to reach a positive response. The stimulus has to picture a male person, who is also an adult and 
on top of that handsome as well, requiring the processing of at least three information pieces. Processing more information requires more time, yielding longer response times for sexually preferred target categories. Hence, VT effects are concomitant with a feature-checking process that is a mere function of what the task determines to do with the targets.

In support of such cold cognitive task byproducts, Imhoff and colleagues (2012) showed that heterosexual men completing a standard VT task from the assigned vicarious perspective of a gay man ("How attractive is this person to gay men?") exhibited response time patterns mimicking that of gay men. Likewise, gay men responding from an assigned heterosexual perspective had response time patterns resembling that of straight men. In contrast, assigning perspectives that matched participants' sexual orientations yielded the expected sexual orientation-congruent VT effects. This is well compatible with the notion that the cascade of cognitive processes necessary to perform the judgement task (which is independent of the sexual orientation of the responder) is responsible for these response time patterns (i.e., a task effect) rather than a reaction that the stimuli themselves evoke in the responder (i.e., a stimulus effect). Further support for this feature-checking account comes from the fact that rating the sex rather than the sexual attractiveness of the depicted person eradicated response time differences between gay and straight men in VT tasks (Pohl et al., 2016).

\section{Hot Stimulus-Based Reactions}

Despite this initial support for the relevance of cold cognitive processes stemming from task effects, hot responses that actually reflect a form of stimulus-driven sexual responding may still play a role. For example, besides a large task effect on response times in the studies by Imhoff et al. (2012), they also observed an independent but less pronounced interaction effect of participant sexual orientation and target sex independent of the assigned perspectives. That is, on 
top of the task demands, heterosexual men still had slightly longer response times for trials with female stimuli and gay men had slightly longer response times for male stimuli. The stimuli may thus still evoke a kind of sexual content induced delay (SCID) that is currently not well understood. Such a pattern has also been reported for attentional cueing paradigms where a SCID-like longer response was robustly correlated with individual differences in dispositional sexual excitation (Imhoff et al., 2020). Crucially, this dovetails with the notion that VT paradigms without any rating task (i.e., free picture viewing tasks sometimes used prior to classic VT rating tasks in order to familiarize participants with the stimulus material) also might reveal participants' sexual interests. Based on the stimuli's inherent attention-grabbing potential, it is supposed that the differential sexual appeal of the presented stimuli is capable to trigger hot decision processes that evoke sexual orientation-specific response time patterns. To date, however, there is not much empirical support for this VT task variant except from a study by Bourke and Gormley (2012) and an early VT set-up that had been used by Zamansky (1956).

\section{Present Research}

We conducted an experimental study to test the causal role of task demands and hot stimulus-based processes in VT set-ups. Specifically, we reasoned that stimulus-based hot processes would imply the predictions a) that VT patterns emerge also when respondents merely watch the stimuli without having to evaluate them, b) that VT effects are more pronounced for sexually more suggestive (i.e., naked) stimuli, and c) that the extent of VT effects are systematically related to self-report measures of dispositional sex drive. Specifically, sex drive should increase the target sex specificity of sexual preferences. However, this should be more pronounced for males than for females due to the increased target sex non-specificity of heterosexual females' sexual interests (Lippa, 2006, 2017; Chivers, 2017). Conversely, task- 
demands a) should not create any differences between respondents with different sexual orientations if there was no rating task involved and b) should yield a pattern akin to typical VT effects even in the complete absence of any sexually suggestive pictorial stimuli as long as the cognitive demands of the task are similar (i.e., as exemplified by stimuli that represent nonpictorial, merely symbolical representations of stimulus features such as target sex, age, and attractiveness). Moreover, we supposed c) VT sexual preference scores would lack associations with dispositional sex drive indicators if task demands were the causal drivers of VT effects. Thus, participants worked through three different VT paradigms (VT without rating task, standard VT task; symbolic VT task) that allowed us to test both accounts' predictions across a variety of outcomes.

\section{Method}

\section{Participants}

A student sample of 145 individuals ( 75 females) from a German university participated in an experiment on the perception of human individuals for course credit. Participants were informed that partaking was voluntary and that they could quit the experiment at any time without any disadvantage. They were told that the study explored the underlying mechanisms of perceiving and processing human adults and children. Apart from this, they did not receive any further information about the content of the experiment to avoid creating a context that implied the assessment of sexual interests. Nine participants were excluded due to their selfcategorization subsequent to all VT assessments as not exclusively or mostly heterosexual (i.e., Kinsey scale $>1$, see below). The final sample thus consisted of $N=136$ participants (67 females; $49.3 \%)$. Age $(M=22.9, S D=4.4$ years $)$ ranged between 16 and 39 years with males 
being on average roughly two years older, $t(128.217)=2.739, p=.007, d=0.47$ and females, expectedly, revealing less exclusively heterosexual orientations $\left(\chi^{2}(1)=7.278, p=.007, \phi=.23\right)$.

\section{Measures and Procedure}

After having obtained informed consent from the participants they were randomized into one of four experimental conditions. According to a 2 (Participant Instruction) x 2 (Target Nudity) between-subjects factorial design participants received either an instruction before the first VT task mentioning that the present study is about sexual interests and sexuality or no further instruction. This experimental manipulation was added because we sought to test whether the VT effects without rating tasks reported in Bourke and Gormley (2012) could be explained by the fact that their study procedure had made sexual content salient before assessing VT (i.e., by assessing sexual orientation and for some participants other indirect latency-based paradigms involving explicit processing of sexual attractiveness). Additionally, target stimuli were either presented nude or in bathing clothes in the two following pictorial viewing tasks to manipulate sexual suggestiveness. All stimuli throughout the whole experiment were presented in a prefixed randomized order for all participants in order to keep possible position and order effects constant and to be able to attribute variability in response times to person effects.

\section{Viewing Time Task Without Ratings}

Before the first VT task commenced, all participants were instructed that they will see pictures as shown during the further study and that they could skip to the next picture using the answer key on the right of the computer keyboard. Notably, no further instructions to rate pictures in terms of any characteristic followed. This initial VT task without rating instructions consisted of two practice trials (pictures of one male and one female adult). These were followed by 16 test trials of each four-according to the respective experimental condition clothed or nude- 
male and female adult stimuli equally distributed over Tanner stages 4 and $5^{1}$ from the Not-Real People picture set (subset A; Pacific Psychological Assessment Corporation, 2004) that had not been used in the practice trials. This frequently employed picture set contains two parallel sets of assembled pictures of non-existing female and male humans (nude or in bathing clothes) across varying physical maturation stages. Response latencies for every picture were recorded unobtrusively while stimuli were presented in the center of the computer screen until participants decided when to move on to the next picture by pressing the answer key.

\section{Standard Viewing Time Task}

Subsequently, participants completed a standard VT paradigm (Imhoff et al., 2010, Study 1) without restriction of response times where they were explicitly instructed to rate the subjective sexual attractiveness of the presented stimuli from their own perspective on a Likert scale from 1 (sexually unattractive) to 5 (sexually attractive). Again, stimuli were presented nude or clothed according to the experimental condition participants had been assigned to. Paralleling the first task, the second VT task consisted of 16 new ( +2 practice) trials with equal amounts of male and female adult stimuli from Tanner stages 4 and 5 of the Not-Real People picture set (subset B; Pacific Psychological Assessment Corporation, 2004). Stimuli were presented in the center of the screen with the Likert scale for the sexual attractiveness ratings positioned underneath. Participants had to press the corresponding answer keys from 1 to 5 on the keyboard followed by hitting the enter key while response latencies (from stimulus onset until pressing enter) for each trial were recorded unobtrusively.

Individual Differences in Sex Drive and Control Variables

\footnotetext{
${ }^{1}$ Tanner stages (Tanner, 1978) represent physical indicators of sexual maturation (i.e., pubertal status). Tanner stages 4 and 5 depict postpubescent individuals.
} 
The standard VT task was followed by a short filler task that was used to assess sex driverelated control variables. These consisted of the five-item Sexual Preoccupation scale taken from Wiedermann and Allgeier's (1993) shortened Sexuality Scale (German version by Brenk-Franz and Strauß, 2011; e.g., "I think about sex more than anything else"; $\alpha=.89$ ). Items were rated on a five-point Likert scale ranging from 1 (does not apply at all to me) to 5 (does fully apply to me). These were followed by four single items tapping into the total number of sexual outlets per week, the subjectively perceived strength of sexual desire, the average number of minutes spent on sexual fantasizing per day (all over the last four weeks) and the time interval since participants' last sexual activity including masturbation from 0 (a few hours) to 5 (more than a week).

Symbolic Viewing Time Task

After this, a newly developed symbolic VT task followed. In contrast to the depictions of full human bodies (or larger parts of them) in the first two VT tasks, all stimuli in the symbolic VT task variant were non-pictorial (i.e., did not depict bodies or body parts) consisting only of abstract symbols that represented meaningful target characteristics. Based on the featurechecking hypothesis implying that (at least) target sex, age, and attractiveness need to be checked to verify stimulus sexual attractiveness (Imhoff et al., 2010; 2012), we used symbols that depicted these three target characteristics. Hence, each symbol representing a single target stimulus consisted of (1) a letter indicating target sex ("M" for male [German männlich], "W"construed as a $180^{\circ}$-rotation of the male $\mathrm{M}$ symbol- for female [German weiblich]), (2) a number representing target age $(10,12,20$, or 22 years), and (3) a “+” or “_-“ sign depicting sexy or unsexy target stimuli, respectively. Age and attractiveness indicators were embedded into the target sex symbols (see Figure 1 for example stimuli). We counterbalanced whether the 
attractiveness indicator was positioned above or below the age indicator across all 32 test trials in the symbolic VT task. Prior to the test trials, four practice trials (one male and female child [11 years] and adult stimulus [21 years] each, with adults being unattractive and children being attractive) had been presented. Utilizing stimulus examples, the meaning of each single indicator composing the whole symbolic representation of a target stimulus was explained to the participants on a single instruction screen where they also were shown how to rate whether the target symbols represented a sexually attractive sex partner or not. Again, response decisions were recorded unobtrusively without response time restrictions via two red answer keys on the computer keyboard (left and right for sexually unattractive and attractive partners, respectively).

\section{(insert Figure 1 about here)}

\section{Demographics and Sexual Orientation}

The study closed with two demographic items (participant sex and age) and the Kinsey scale. On the latter participants positioned their sexual orientation (explicitly referring not only to previous behavior but also fantasies) on a scale from 0 (exclusively heterosexual) to 6 (exclusively homosexual) including a response option to indicate complete lack of sexual desire and contact (Kinsey, Pomeroy, \& Martin, 1948; Kinsey, Pomeroy, Martin, \& Gebhard, 1953).

\section{Results}

\section{Data Preparation and Control Analyses}

In accordance with prior VT literature (e.g., Imhoff et al., 2010; 2012), reaction times below 300ms were discarded (VT without rating: 5 trials [0.2 \%]; standard VT: 0 trials; symbolic VT: 9 trials $[0.2 \%]$ ), and latencies $>10.000 \mathrm{~ms}$ were truncated (VT without rating: 7 trials $[0.3$ \%]; standard VT: 27 trials [1.2\%]; symbolic VT: 11 trials [0.3\%]) for all analyses involving response latencies as dependent variables. Except from the selection of participants according to 
their exclusivity of heterosexual orientation (as described in the Participants section) no further filters of participants or modifications of the raw data have been implemented.

Control analyses $\left(\chi^{2}\right.$-tests, Oneway-ANOVAs) revealed that the four experimental groups did not differ in terms of participant sex distribution, $\chi^{2}(3)<1, p=.986, \phi=.03$, and exclusivity of heterosexual orientation, $\chi^{2}(3)=6.430, p=.092, \phi=.22$. Moreover, no systematic group differences emerged concerning age, $F(3,132)<1, p=.634, \eta^{2}=.013$, self-reported sexual preoccupation, $F(3,132)<1, p=.463, \eta^{2}=.019$, total sexual outlets/week, $F(3,71.832)=1.610$, $p=.195, \eta^{2}=.068$, daily time spent on sexual fantasizing, $F(3,64.417)=1.608, p=.196, \eta^{2}=$ .023 , subjectively perceived strength of sexual desire, $F(3,132)<1, p=.396, \eta^{2}=.022$, and time since last sexual activity, $F(3,127)<1, p=.786, \eta^{2}=.008$.

\section{Viewing Time Task Without Rating}

In order to test whether basic VT effects emerged in the free picture viewing task without the sexual attractiveness rating task we conducted a 2 (Participant Sex) x 2 (Participant Instruction) x 2 (Target Nudity) x 2 (Target Sex) mixed-model ANOVA with the first three factors having been manipulated between subjects and mean target category response latencies as dependent variables. With the exception of a significant main effect of Target Sex as exemplified by moderately slower responses to female adult stimuli (Figure 2), no further significant main or interaction effects were observed (Table 1). In the VT task without explicit sexual attractiveness rating instructions, the Target Sex x Participant Sex interaction constituting the crucial diagnostic VT effect failed to reach significance. Importantly, this basic VT effect also was not further qualified by the experimental manipulations of target nudity and participant instructions of the study context as corroborated by the lack of any higher-order interactions. Post-hoc tests revealed that female participants viewed female targets $(M=1852 \mathrm{~ms}, S D=1037)$ slightly longer 
than male targets $(M=1782 \mathrm{~ms}, S D=966), t(66)=2.005, p=.049, d_{\mathrm{z}}=0.08$, whereas male participants' response latencies did not differ between female $(M=2215 \mathrm{~ms}, S D=1526)$ and male targets $(M=2088 \mathrm{~ms}, S D=1544), t(68)=1.704, p=.093, d_{z}=0.08$.

(insert Figure 2 and Table 1 about here)

\section{Standard Viewing Time Task}

Verifying participants' self-reported heterosexual orientation as well as testing the impact of nude stimuli on a standard VT paradigm, we ran a 2 (Participant Sex) x 2 (Target Sex) x 2 (Target Nudity) mixed-model ANOVA with the first factor being manipulated between-subjects. Again, mean target category response latencies served as the dependent variables. The only effects that yielded significant results (Table 2) were the main effect of Target Sex (i.e., longer latencies for female than for male targets) which was further qualified by the interaction with Participant Sex replicating the theoretically expected heterosexual orientation pattern (Figure 3). Post-hoc tests revealed that heterosexual females showed longer response times for adult male stimuli $(M=3336 \mathrm{~ms}, S D=1187)$ than for adult female stimuli $(M=3115 \mathrm{~ms}, S D=1183), t(66)$ $=2.190, p=.032, d_{\mathrm{z}}=0.19$, and heterosexual males yielded longer response times for adult female stimuli $(M=4064 \mathrm{~ms}, S D=1816)$ than for adult male stimuli $(M=2659 \mathrm{~ms}, S D=1504)$, $t(68)=9.205, p<.001, d_{z}=0.83$. Notably, Target Nudity did not reveal any statistically significant main or interaction effect on VT latencies (Table 2).

(insert Table 2 and Figure 3 about here)

\section{Symbolic Viewing Time Task}

Within the symbolic VT task we first explored whether participants had understood and correctly followed the instructions by testing whether their responses (not response times) reflected normatively valid heterosexual orientation patterns. To this end, we conducted a 2 
(Participant Sex) x 2 (Target Sex) x 2 (Target Maturity) x 2 (Target Attractiveness) mixed-model ANOVA with the first factor varying between-subjects and mean target category sexual attractiveness ratings as dependent variables. As revealed by the significant full four-way interaction of all factors (Table 3), participants produced theoretically meaningful decision patterns in the symbolic VT task: All participants preferred adult over child targets and attractive over unattractive targets as exemplified by the corresponding significant main effects (Table 3 ). These maturity and attractiveness preferences interacted with Target Sex and Participant Sex (Table 3) as participants found targets of the opposite sex sexually more appealing than same-sex targets. This pattern was more pronounced for attractive over unattractive targets and for adults over children (Figure 4). Post-hoc tests of the symbolic ratings revealed large sexual orientation effects for female participants who rated adult males $(M=.46, S D=.25)$ as sexually more attractive than adult females $(M=.13, S D=.25), t(66)=9.449, p<.001, d_{z}=1.23$. Male participants showed the opposite pattern for adult females $(M=.61, S D=.23)$ over adult males $(M=.05, S D=.16), t(68)=16.015, p<.001, d_{z}=3.79$.

\section{(insert Table 3 and Figure 4 about here)}

Given the fact that participants had performed the sexual attractiveness ratings in the symbolic VT task according to the instructions, we reran the same mixed-model ANOVA as for the sexual attractiveness ratings with target category response latencies as dependent variables. Table 3 gives an overview of the ANOVA results. Mimicking a classic viewing time pattern, the crucial three-way interaction of Participant Sex, Target Sex, and Target Maturity was significant and in accordance with participants' heterosexual orientation. Female participants showed longer response times for male than for female targets and male participants vice versa. This pattern was more pronounced for adult than for child targets (Figure 5; see Figure A in the Electronical 
Supplement for full factorial results of the response latency ANOVA including the two significant but theoretically less relevant three-way interactions that lacked either the influences of Target Maturity or Target Sex which are crucial determinants for teleiophilic heterosexual orientations). Post-hoc tests confirmed that in the symbolic VT task, female participants showed faster response latencies for adult females $(M=1559 \mathrm{~ms}, S D=715)$ than for adult males $(M=$ $1956 \mathrm{~ms}, S D=882), t(66)=4.544, p<.001, d_{\mathrm{z}}=0.49$, and for female child stimuli $(M=1155 \mathrm{~ms}$, $S D=547)$ than for male child stimuli $(M=1412 \mathrm{~ms}, S D=521), t(66)=4.063, p<.001, d_{z}=$ 0.48 . Male participants showed the opposite pattern for female $(M=2189 \mathrm{~ms}, S D=1089)$ and male adult stimuli $(M=1321 \mathrm{~ms}, S D=595), t(68)=9.227, p<.001, d_{z}=0.94$, but no statistically significant difference between female $(M=1479 \mathrm{~ms}, S D=540)$ over male $(M=$ $1406 \mathrm{~ms}, S D=573)$ child stimuli, $t(68)=1.136, p=.260, d_{\mathrm{z}}=0.13$.

For the sake of completeness, it should be noted that the results yielded no support for the full four-way interaction of Participant Sex, Target Sex, Target Maturity, and Target Attractiveness $\left(p=.327, \eta_{\mathrm{p}}^{2}=.007\right)$ that was found for the attractiveness ratings. Importantly, however, this interaction is not predicted from a feature-checking perspective as knowing that a target has the right age and sex, but is not attractive should not be faster than knowing that the target has the right age, sex and is attractive (as a binary decision for attractive or not can be reached in an identical time independent of whether the answer is yes or no).

$$
\text { (insert Figure } 5 \text { about here) }
$$

\section{Reliability, Validity, and Sex Drive Correlations}

The standard VT sexual preference scores for adult targets (i.e., ratings/latencies for female target categories minus ratings/latencies for male target categories) showed at least sufficient internal consistency (ratings $>$ latencies, Table 4). The symbolic VT yielded similarly reliable 
results in case of the latency-based preference score whereas for the preference score based on explicit ratings reliability was attenuated. Notably, the VT task without any rating instruction produced a markedly unreliable difference score, arguably because it did not measure sexual preference (Table 4).

In terms of convergent validities, VT sexual preference scores based on ratings and latencies showed large within-task correlations $(r \mathrm{~s}>=.63)$ and were strongly associated with participant sex as a proxy for sexual orientation $(r \mathrm{~s}>=.61$; Table 4$)$. All other intercorrelations were also substantial thereby corroborating the validity of all VT tasks that included stimulus ratings. In contrast, the VT task without rating was unrelated to participant sex or any other VT sexual preference score in this study. This finding attests to the notion that the rating task is indeed necessary for VT tasks to be diagnostic of sexual interests.

Intercorrelations of sex drive indicators and sexual preference scores revealed that latencybased and explicitly-rated sexual preference scores were not significantly associated with any sex drive indicator tested here except for the VT symbolic rating preference score and self-reported total sexual outlets (Kendall's $\tau=.23$; Table 4). However, accounting for multiple testing this single significant association (out of 50 sex drive correlations) is likely due to chance and, thus, needs to be regarded with caution.

(insert Table 4 about here)

\section{Discussion}

\section{Cold Task-Dependent Versus Hot Stimulus-Driven Processes}

The current results provide consistent support for the feature-checking account of VT effects. Participants' decision latencies mimicked standard VT patterns (including the well documented lesser target sex specificity of heterosexual females' sexual interests; Chivers, 2017; 
Lippa, 2017) even when they rated stimuli that were devoid of any pictorial sexual connotations while the rating process still required the same cognitive processing of the three relevant features proposed by the feature-checking hypothesis (i.e., target sex, age, and attractiveness). In contrast, the results did not provide any convincing support for hot processes involved in actual sexual responding: There were no theoretically meaningful VT patterns observed in the absence of a rating task. Moreover, naked stimuli did not pronounce standard VT effects and systematic correlations between VT sexual preference scores and sex drive measures were missing.

In summary, the findings provide strong evidence that prolonged VT response latencies for sexually preferred target stimuli are caused by a cold cognitive process. The results underscore that VT effects do not rely on attentional phenomena and do not tap into physiological sexual arousal. Instead, VT effects are a byproduct of the fact that determining sexual attractiveness takes longer (because more features-here target sex, age, and attractiveness-need to be checked and verified) than ruling it out by simply rejecting one basic feature. Although we cannot definitively rule out the involvement of any hot process of sexual responding, arguably these were too small to be detected within the current experiment.

\section{Limitations and Outlook}

While our study is limited in that it is based on a single experiment involving solely heterosexual (highly likely teleiophilic) community participants, its results are particularly strong and unidirectional. At present, it seems unlikely that the cognitive architecture of the VT task would be drastically different for other samples involving more diverse sexual orientations as implied by concomitant findings for gay males (Imhoff et al., 2010; 2012; Pohl et al., 2016). It might be conceivable, however, that the very nature of a computer-guided experimental task such as the current one, brings cognitive task demands to the forefront and masks sexual 
responding that would be expected in more naturalistic settings or in states of experimentally induced acute sexual arousal (Imhoff $\&$ Schmidt, 2014). While this might be interesting from a basic research perspective, the current experiment arguably is a better representation of the diagnostic settings that involve VT assessments than an experiment with a sexual arousal induction would. In applied settings, VT measures are primarily used in forensic contexts where the assessment of sexual interests in individuals who have sexually offended against children is of high relevance for risk management purposes and where the non-invasiveness of these measures is a welcome surplus (Schmidt \& Perkins, 2020).

Our findings add another piece to the puzzle of indirect latency-based sexual interest diagnostics. Practitioners and researchers are confronted with the fascinating conundrum that VT tasks are driven by cold cognitive processes unrelated to sexual responding in the narrow sense, but are still valid proxies for actual sexual interest as long as respondents truthfully and authentically categorize the presented stimuli according to their subjectively perceived sexual attractiveness. Notably, it is exactly this task-dependency that poses a diagnostic liability to VT assessments as merely switching task demands renders these measures invalid for sexual interest assessments (Imhoff et al., 2012; Pohl et al., 2016; see also the non-diagnosticity of the VT task without rating in the present research). It has to be kept in mind, though, that task compliance is a necessary prerequisite for the validity of most psychological measures in any construct domain. Therefore, VT tasks provide no magic bullet to tap into hidden or "implicit" sexual orientations but merely provide an additional, non-verbal (i.e., indirect) pathway to participants' responses (Schmidt \& Banse, in press). Nevertheless such measures show incremental validity above and beyond self-reported sexual interest in children and adults (Banse et al., 2010). The present findings may allow the conclusion that this is not due to their reflection of "unconscious" 
orientations or the ability to effectively rule out social desirable faking, but because any valid additional measure that does not share method variance with self-reports will result in a more accurate estimate of the underlying construct.

In conclusion, the present study elucidates the cognitive architecture that underlies the VT effect and thereby emphasizes that the term viewing time itself represents a misnomer. Instead of using VT or the merely descriptive "prolonged response latencies for sexually attractive targets (PRELSAT)" (Imhoff et al., 2010, p. 1279), scholars may legitimately refer to the measure as a decision time measure. At the very least, researchers and practitioners should keep in mind the underlying processes that produce the measurement outcome in order not to have unrealistic hopes regarding its nature of a bona fide pipeline to unconscious sexual desires.

\section{Compliance with Ethical Standards}

\section{Conflict of Interest}

The authors declare that they have no conflict of interest.

\section{Research involving Human Participants and/or Animals}

All procedures performed in this study involving human participants were in accordance with the ethical standards of the institutional and/or national research committee and with the 1964 Helsinki declaration and its later amendments or comparable ethical standards. This article does not contain any studies with animals performed by any of the authors.

\section{Informed consent}

Informed consent was obtained from all individual participants included in the study. 


\section{References}

Banse, R., Schmidt, A. F., \& Clarbour, J. (2010). Indirect measures of sexual interest in child sex offenders: A multi-method approach. Criminal Justice and Behavior, 37, 319-335. https://doi.org/10.1177\%2F0093854809357598

Brenk-Franz, K., \& Strauß, B. (2011). Der Multidimensionale Fragebogen zur Sexualität (MFS). Zeitschrift für Sexualforschung, 24(3), 256-271. https://doi.org/10.1055/s-0031-128706

Bourke, A. B., \& Gormley, M. J. (2012). Comparing a pictorial stroop task to viewing time measures of sexual interest. Sexual Abuse, 24(5), 479-500. https://doi.org/10.1177/1079063212438922

Chivers, M. L. (2017). The specificity of women's sexual response and its relationship with sexual orientations: A review and ten hypotheses. Archives of Sexual Behavior, 46(5), 1161-1179. https://doi.org/10.1007/s10508-016-0897-x

Dawson, S. J., Suschinsky, K. D., \& Lalumiere, M. L. (2012). Sexual fantasies and viewing times across the menstrual cycle: A diary study. Archives of Sexual Behavior, 41(1), 173183. https://doi.org/10.1007/s10508-012-9939-1

Ebsworth, M., \& Lalumière, M. L. (2012). Viewing time as a measure of bisexual sexual interest. Archives of Sexual Behavior, 41(1), 161-172. https://doi.org/10.1007/s10508-012$\underline{9923}$

Gray, S. R., Abel, G. G., Jordan, A., Garby, T., Wiegel, M., \& Harlow, N. (2015). Visual Reaction Time ${ }^{\mathrm{TM}}$ as a predictor of sexual offense recidivism. Sexual Abuse, 27(2), 173188. https://doi.org/10.1177/1079063213502680

Harris, G. T., Rice, M. E., Quinsey, V. L., \& Chaplin, T. C. (1996). Viewing time as a measure of sexual interest among child molesters and normal heterosexual men. Behaviour 
Research and Therapy, 34(4), 389-394. https://doi.org/10.1016/0005-7967(95)00070-4

Imhoff, R., Barker, P., \& Schmidt, A. F. (2020). To what extent do erotic images elicit visuospatial versus cognitive attentional processes? Consistent support for a (non-spatial) Sexual Content-Induced Delay. Archives of Sexual Behavior, 49(2), 531-550. https://doi.org/10.1007/s10508-019-01512-0

Imhoff, R., \& Schmidt, A. F. (2014). Sexual disinhibition under sexual arousal: Evidence for domain specificity in men and women. Archives of Sexual Behavior, 43(6), 1123-1136. https://doi.org/10.1007/s10508-014-0329-8

Imhoff, R., Schmidt, A. F., Nordsiek, U., Luzar, C., Young, A. W., \& Banse, R. (2010). Viewing time effects revisited: Prolonged response latencies for sexually attractive targets under restricted task conditions. Archives of Sexual Behavior, 39(6), 1275-1288.

https://doi.org/10.1007/s10508-009-9595-2

Imhoff, R., Schmidt, A. F., Weiß, S., Young, A. W., \& Banse, R. (2012). Vicarious viewing time: Prolonged response latencies for sexually attractive targets as a function of task-or stimulus-specific processing. Archives of Sexual Behavior, 41(6), 1389-1401. https://doi.org/10.1007/s10508-011-9879-1

Israel, E., \& Strassberg, D. S. (2009). Viewing time as an objective measure of sexual interest in heterosexual men and women. Archives of Sexual Behavior, 38(4), 551-558. https://doi.org/10.1007/s10508-007-9246-4

Jahnke, S., Schmidt, A. F., Klöckner, A., \& Hoyer, J. (2021). A critical examination of neurodevelopmental differences, pedohebephilia, and sexual offending: Findings from two online surveys. Manuscript submitted for publication.

Kalmus, E., \& Beech, A. R. (2005). Forensic assessment of sexual interest: A review. Aggression 
and Violent Behavior, 10(2), 193-217. https://doi.org/10.1016/j.avb.2003.12.002

Kinsey, A. C., Pomeroy, W. B., \& Martin, C. E. (1948). Sexual behavior in the human male. Philadelphia: Saunders.

Kinsey, A. C., Pomeroy, W. B., Martin, C. E., \& Gebhard, P. H. (1953). Sexual behavior in the human female. Philadelphia: Saunders.

Larue, D., Schmidt, A. F., Imhoff, R., Eggers, K., Schönbrodt, F. D., \& Banse, R. (2014). Validation of direct and indirect measures of preference for sexualized violence. Psychological Assessment, 26(4), 1173-1183. https://doi.org/doi/10.1037/pas0000016

Lippa, R. A. (2017). Category specificity of self-reported sexual attraction and viewing times to male and female models in a large US sample: Sex, sexual orientation, and demographic effects. Archives of Sexual Behavior, 46(1), 167-178. https://doi.org/10.1007/s10508-016$\underline{0844-\mathrm{x}}$

Lippa, R. A. (2006). Is high sex drive associated with increased sexual attraction to both sexes? Psychological Science, 17, 46-52.https://doi.org/10.1111/j.1467-9280.2005.01663.x

Oberlader, V. A., Ettinger, U., Banse, R., \& Schmidt, A. F. (2017). Development of a cued proand antisaccade paradigm: An indirect measure to explore automatic components of sexual interest. Archives of Sexual Behavior, 46(8), 2377-2388. https://doi.org/10.1007/s10508$\underline{016-0839-7}$

Pacific Psychological Assessment Cooperation. (2004). The Not-Real People stimulus set for assessment of sexual interest. Author.

Pedneault, C. I., Hilgard, J., Pettersen, C., Hermann, C. A., White, K., \& Nunes, K. L. (2021). How well do indirect measures assess sexual interest in children? A meta-analysis. Journal of Consulting and Clinical Psychology. Advance online publication. 


\section{http://dx.doi.org/10.1037/ccp0000627}

Petterson, L. J., Dixson, B. J., Little, A. C., \& Vasey, P. L. (2018). Viewing time and self-report measures of sexual attraction in Samoan cisgender and transgender androphilic males. Archives of Sexual Behavior, 47(8), 2427-2434. https://doi.org/10.1007/s10508-018-1267$\underline{7}$

Pohl, A., Wolters, A., \& Ponseti, J. (2016). Investigating the task dependency of viewing time effects. The Journal of Sex Research, 53(8), 1027-1035. https://doi.org/10.1080/00224499.2015.1089429

Quinsey, V. L., Ketsetzis, M., Earls, C., \& Karamanoukian, A. (1996). Viewing time as a measure of sexual interest. Ethology and Sociobiology, 17(5), 341-354.

https://doi.org/10.1016/S0162-3095(96)00060-X

Rönspies, J., Schmidt, A. F., Melnikova, A., Krumova, R., Zolfagari, A., \& Banse, R. (2015). Indirect measurement of sexual orientation: Comparison of the implicit relational assessment procedure, viewing time, and choice reaction time tasks. Archives of Sexual Behavior, 44(5), 1483-1492. https://doi.org/10.1007/s10508-014-0473-1

Schmidt, A. F., Babchishin, K. M., \& Lehmann, R. J. (2017). A meta-analysis of viewing time measures of sexual interest in children. Archives of Sexual Behavior, 46(1), 287-300. https://doi.org/10.1007/s10508-016-0806-3

Schmidt, A. F., \& Banse, R. (in press). Using indirect measures of sexual interest in forensic contexts - Past, present, and future. In K. Uzieblo, W. Smid, \& K. McCartan (Eds.), Sex offender assessment, treatment, and management: Emerging directions and debates. Palgrave-Macmillan.

Schmidt, A. F., \& Perkins, D. (2020). Using the Explicit and Implicit Sexual Interest Profile in 
applied forensic or clinical contexts. In G. Ackerman, D. Perkins, \& R. M. Bartels (Eds.), Assessing and managing problematic sexual interests - A practitioner's guide (pp. 57-81). Routledge. http://dx.doi.org/10.4324/9780429287695-4

Tanner, J. (1978). Foetus into man: Physical growth from conception to maturity. Harvard University Press.

Wiederman, M. W., \& Allgeier, E. R. (1993). The measurement of sexual-esteem: Investigation of Snell and Papini' s (1989) Sexuality Scale. Journal of Research in Personality, 27(1), 88-102. https://doi.org/10.1006/jrpe.1993.1006

Xu, Y., Rahman, Q., \& Zheng, Y. (2017). Gender-specificity in viewing time among heterosexual women. Archives of Sexual Behavior, 46(5), 1361-1374. https://doi.org/10.1007/s10508-016-0795-2

Zamansky, H. S. (1956). A technique for assessing homosexual tendencies. Journal of Personality, 24(4), 436-448. https://doi.org/10.1111/j.1467-6494.1956.tb01280.x 


\section{Tables}

\section{Table 1}

Analysis of Variance (ANOVA) for response latencies as a function of Participant Sex, Participant Instruction, Target Nudity, and Target Sex in viewing time task without rating instructions.

\begin{tabular}{|c|c|c|c|c|}
\hline Source & $d f$ & $F$ & $p$ & $\eta_{p}^{2}$ \\
\hline \multicolumn{5}{|l|}{ Between-subjects } \\
\hline Participant Sex (PS) & 1 & 2.301 & .132 & .018 \\
\hline Participant Instruction (I) & 1 & $<1$ & .816 & $<.001$ \\
\hline Target Nudity (TN) & 1 & $<1$ & .891 & $<.001$ \\
\hline PS $x$ PI & 1 & 1.841 & .177 & .014 \\
\hline $\mathrm{PS} \times \mathrm{TN}$ & 1 & $<1$ & .335 & .007 \\
\hline PIx TN & 1 & $<1$ & .532 & .003 \\
\hline PS x PI x TN & 1 & $<1$ & .756 & .001 \\
\hline Within-group error & $128(3,323,050.973)$ & & & \\
\hline \multicolumn{5}{|l|}{ Within-subjects } \\
\hline Target Sex (TS) & 1 & 5.564 & $.020^{*}$ & .042 \\
\hline TS x PS & 1 & $<1$ & .643 & .002 \\
\hline $\mathrm{TS} \times \mathrm{TN}$ & 1 & $<1$ & .682 & .001 \\
\hline TS x PI & 1 & $<1$ & .923 & $<.001$ \\
\hline TS x PS x TN & 1 & $<1$ & .839 & $<.001$ \\
\hline TS x PS x PI & 1 & $<1$ & .838 & $<.001$ \\
\hline TS x TN x PI & 1 & $<1$ & .511 & .003 \\
\hline TS $x$ PS x PI x TN & 1 & 1.166 & .282 & .009 \\
\hline TS within-group error & $128(129,874.059)$ & & & \\
\hline
\end{tabular}

Note. $N=136$. Values in parentheses represent mean square errors. ${ }^{*} p<.05$ 


\section{Table 2}

Analysis of Variance (ANOVA) for response latencies as a function of Participant Sex, Target Nudity, and Target Sex in standard viewing time task.

\begin{tabular}{llrlr}
\hline \multicolumn{1}{c}{ Source } & \multicolumn{1}{c}{$d f$} & $F$ & $p$ & $\eta_{\mathrm{p}}^{2}$ \\
\hline Between-subjects & & & & \\
Participant Sex (PS) & 1 & $<1$ & .560 & .003 \\
Target Nudity (TN) & 1 & $<1$ & .971 & $<.001$ \\
PS x TN & 1 & $<1$ & .465 & .004 \\
Within-group error & $132(3,668,355.630)$ & & & \\
Within-subjects & & & & \\
Target Sex (TS) & 1 & 42.011 & $<.001^{* * *}$ & .241 \\
TS x PS & 1 & 78.601 & $<.001^{* * *}$ & .373 \\
TS x TN & 1 & $<1$ & .820 & $<.001$ \\
TS x PS x TN & 1 & 3.149 & .078 & .023 \\
TS within-group error & $132(570,743.314)$ & & & \\
\hline
\end{tabular}

Note. $N=136$. Values in parentheses represent mean square errors.

${ }^{* *} p<.001$ 
Table 3

Analyses of Variance (ANOVAs) for explicit ratings and response latencies as a function of Participant Sex, Target Sex, Target Maturity, and Target Attractiveness in symbolic viewing time task.

\begin{tabular}{|c|c|c|c|c|c|c|c|}
\hline \multirow{2}{*}{ Source } & \multirow[b]{2}{*}{$d f$} & \multicolumn{3}{|c|}{ Explicit ratings } & \multicolumn{3}{|c|}{ Response latencies } \\
\hline & & $F$ & $p$ & $\eta_{p}^{2}$ & $F$ & $p$ & $\eta_{p}^{2}$ \\
\hline \multicolumn{8}{|l|}{ Between-subjects } \\
\hline Participant Sex (PS) & 1 & $<1$ & .935 & $<.001$ & $<1$ & .351 & .007 \\
\hline Within-group error & $\begin{array}{l}134^{\mathrm{a}} ; 133^{\mathrm{b}} \\
\left(0.190^{\mathrm{a}} ; 2,509,657.830^{\mathrm{b}}\right)\end{array}$ & & & & & & \\
\hline \multicolumn{8}{|l|}{ Within-subjects } \\
\hline Target Sex (TS) & 1 & 26.986 & $<.001^{* * *}$ & .168 & 2.797 & .097 & .021 \\
\hline Target Maturity (TM) & 1 & 497.983 & $<.001^{* * *}$ & .788 & 83.694 & $<.001^{* * *}$ & .386 \\
\hline Target Attractiveness (TA) & 1 & 217.097 & $<.001^{* * *}$ & .618 & 4.970 & $.027^{*}$ & .036 \\
\hline TS $x$ PS & 1 & 330.462 & $<.001^{* * *}$ & .718 & 81.013 & $<.001^{* * *}$ & .379 \\
\hline TM x PS & 1 & 9.504 & $.002^{* *}$ & .066 & 3.344 & .070 & .025 \\
\hline TA $x$ PS & 1 & $<1$ & .779 & .001 & 1.375 & .243 & .010 \\
\hline $\mathrm{TS} \times \mathrm{TM}$ & 1 & 15.514 & $<.001^{* * *}$ & .104 & 23.912 & $<.001^{* * *}$ & .152 \\
\hline TS x TA & 1 & 3.652 & .058 & .027 & 32.349 & $<.001^{* * *}$ & .196 \\
\hline TM x TA & 1 & 226.957 & $<.001^{* * *}$ & .629 & 13.813 & $<.001^{* * *}$ & .094 \\
\hline TS $x$ TM x PS & 1 & 265.748 & $<.001^{* * *}$ & .665 & 48.383 & $<.001^{* * *}$ & .267 \\
\hline TS $x$ TA $x$ PS & 1 & 159.690 & $<.001^{* * *}$ & .544 & 16.467 & $<.001^{* * *}$ & .110 \\
\hline TM $x$ TA $x$ PS & 1 & $<1$ & .563 & .003 & 23.450 & $<.001^{* * *}$ & .150 \\
\hline TS x TM x TA & 1 & 1.801 & .182 & .013 & 1.815 & .180 & .013 \\
\hline TS x TM x TA x PS & 1 & 133.931 & $<.001^{* * *}$ & .500 & $<1$ & .326 & .007 \\
\hline Within-group error & $134^{\mathrm{a}} ; 133^{\mathrm{b}}$ & & & & & & \\
\hline $\mathrm{TS}$ & $\left(0.44^{\mathrm{a}} ; 250,407.282^{\mathrm{b}}\right)$ & & & & & & \\
\hline TM & $\left(0.40^{\mathrm{a}} ; 503,239.559^{\mathrm{b}}\right)$ & & & & & & \\
\hline TA & $\left(0.45^{\mathrm{a}} ; 339,889.065^{\mathrm{b}}\right)$ & & & & & & \\
\hline $\mathrm{TS} \times \mathrm{TM}$ & $\left(0.46^{\mathrm{a}} ; 305,882.098^{\mathrm{b}}\right)$ & & & & & & \\
\hline TS $x$ TA & $\left(0.33^{\mathrm{a}} ; 258,695.409^{\mathrm{b}}\right)$ & & & & & & \\
\hline TM x TA & $\left(0.36^{\mathrm{a}} ; 452,877.970^{\mathrm{b}}\right)$ & & & & & & \\
\hline TS x TM x TA & $\left(0.33^{\mathrm{a}} ; 250,407.282^{\mathrm{b}}\right)$ & & & & & & \\
\hline
\end{tabular}




\section{Table 4}

Reliabilities (Cronbach's $\alpha$ in brackets) and validities of adult sexual preference measures (females over males) in the full sample as well as correlations with sex drive indicators per participant sex.

\begin{tabular}{|c|c|c|c|c|c|}
\hline & 1. & 2. & 3. & 4. & 5. \\
\hline 1. VT Standard (rating) & $(.89)$ & $.61^{* * * *}$ & .13 & $.63_{* * * *}^{* * *}$ & $.43_{* * * *}^{* * *}$ \\
\hline 2. VT Symbolic (rating) & & $(.53)$ & .11 & $.54^{* * *}$ & $.70^{* * *}$ \\
\hline 3. VT without rating (latencies) & & & $(.35)$ & .02 & .17 \\
\hline 4. VT Standard (latencies) & & & & $(.66)$ & $.50^{* * *}$ \\
\hline 5. VT Symbolic (latencies) & & & & & $(.63)$ \\
\hline Participant sex ${ }^{a}$ & $-.65^{* * *}$ & $-.84^{* * *}$ &.-04 & $-.61^{* * *}$ & $-.65^{* * *}$ \\
\hline \multicolumn{6}{|l|}{ Female participants only ${ }^{\mathrm{b}}$} \\
\hline Sexual Preoccupation Scale ${ }^{c}$ & -.05 & .03 & -.02 & .15 & .10 \\
\hline Total sexual outlets/week $^{\mathrm{c}}$ & -.05 & .18 & .04 & -.06 & .11 \\
\hline Sexual desire strength ${ }^{c}$ & -.09 & .10 & -.02 & .07 & .05 \\
\hline Sexual fantasies $/ \mathrm{d}^{\mathrm{c}}$ & -.06 & .03 & -.01 & .05 & .05 \\
\hline Time since last sexual activity ${ }^{\mathrm{d}}$ & .01 & -.08 & -.03 & .12 & -.03 \\
\hline \multicolumn{6}{|l|}{ Male participants onlyb } \\
\hline Sexual Preoccupation Scale ${ }^{\mathrm{e}}$ & .01 & .11 & .08 & -.11 & .04 \\
\hline Total sexual outlets/week $^{\mathrm{e}}$ & .11 & $.23^{*}$ & .13 & .07 & .06 \\
\hline Sexual desire strength ${ }^{\mathrm{e}}$ & .04 & .13 & .15 & -.08 & -.02 \\
\hline Sexual fantasies/d $\mathrm{e}^{\mathrm{e}}$ & .09 & -.10 & .07 & -.16 & -.02 \\
\hline Time since last sexual activity ${ }^{\mathrm{f}}$ & -.09 & .05 & .04 & .04 & -.09 \\
\hline
\end{tabular}

Note. $N=136 .{ }^{a}$ higher scores depict female sex; ${ }^{b}$ non-parametric Kendall $\tau$ correlations ${ }^{\mathrm{c}} n=67 ;{ }^{\mathrm{d}} n=66 ;{ }^{\mathrm{e}} n=69 ;{ }^{\mathrm{f}} n=65$.

${ }^{*} p<.05 ;{ }^{* * *} p<.001$ 


\section{Figures}

\section{Figure 1}

Stimulus examples from the symbolic viewing time task.

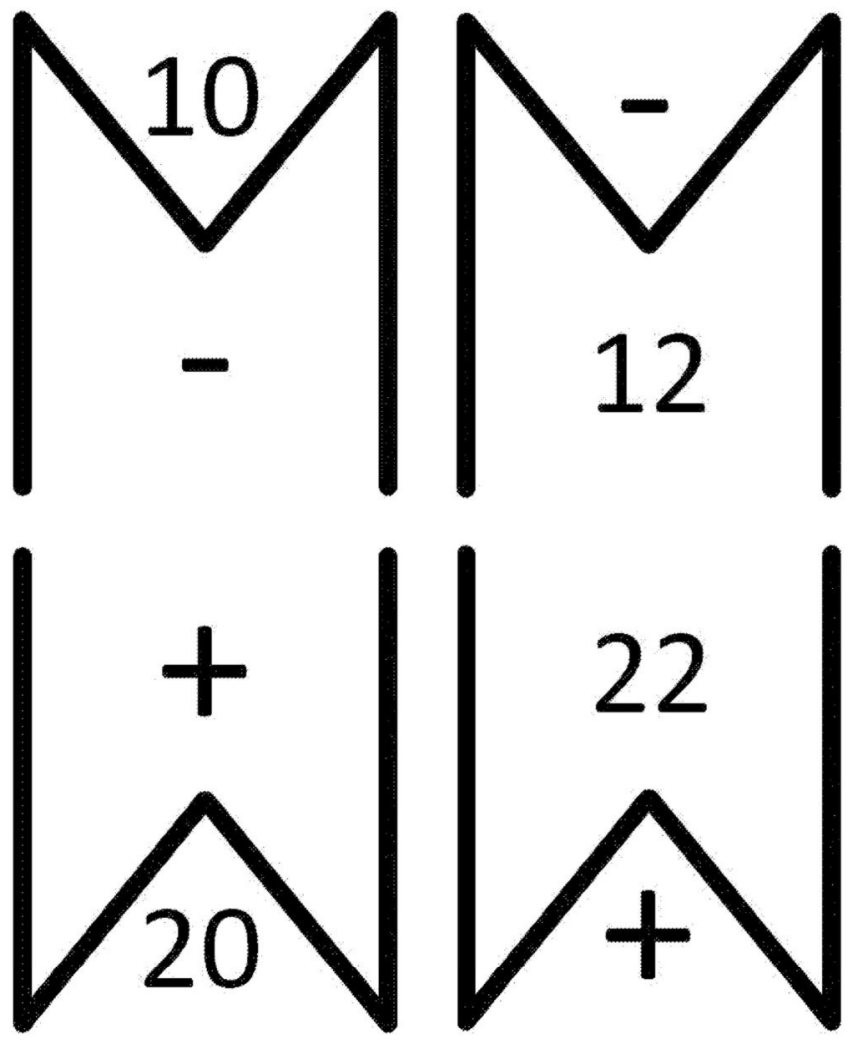

Note. Letters represent stimulus sex $(\mathrm{M}=$ male, German $\boldsymbol{m a ̈ n n l i c h ; ~} \mathrm{W}=$ female, German weiblich). Numbers reflect stimulus age $(10,12,20$, and 22 years of age with the former two indicating children and the latter two adults). Plus and minus signs depict stimulus attractiveness $(+=$ attractive; $-=$ unattractive). Locations of age and attractiveness indicators (upper half/lower half) have been counterbalanced across stimuli. 
Figure 2

Response latencies $\left( \pm C I_{95 \%}\right)$ as a function of Participant and Stimulus Sex in the viewing time task without rating instruction.

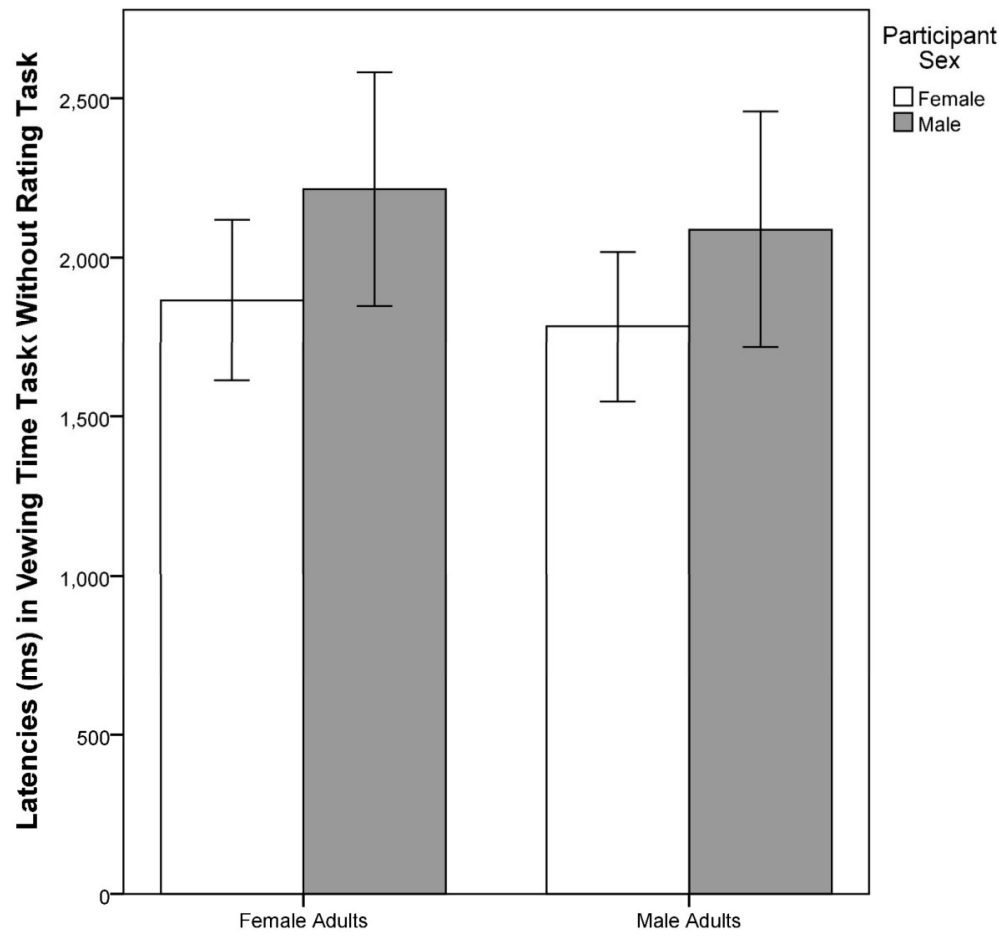


Figure 3

Response latencies ( $\pm C_{95 \%}$ ) as a function of Participant and Stimulus Sex in the standard viewing time task.

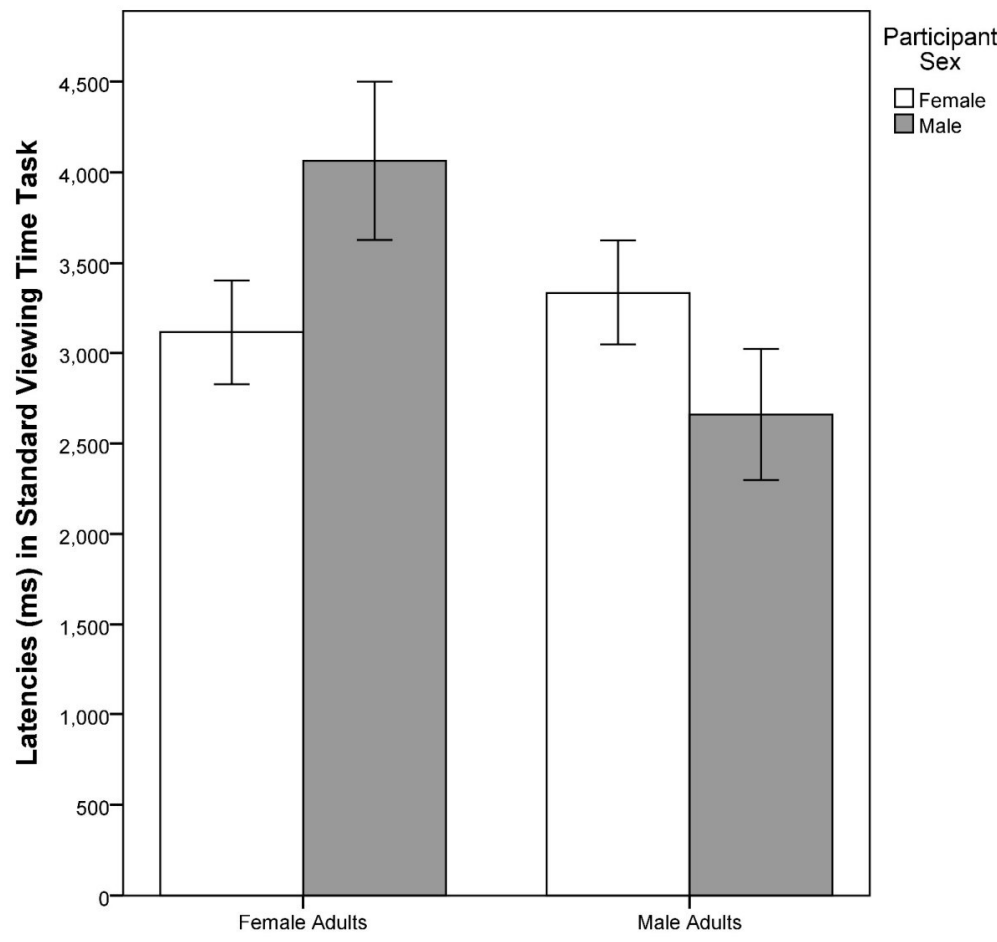


Figure 4

Sexual attractiveness ratings $\left( \pm C I_{95 \%}\right)$ as a function of Participant Sex, Stimulus Sex, Stimulus Sexual Maturity, and Stimulus Attractiveness in the symbolic viewing time task.

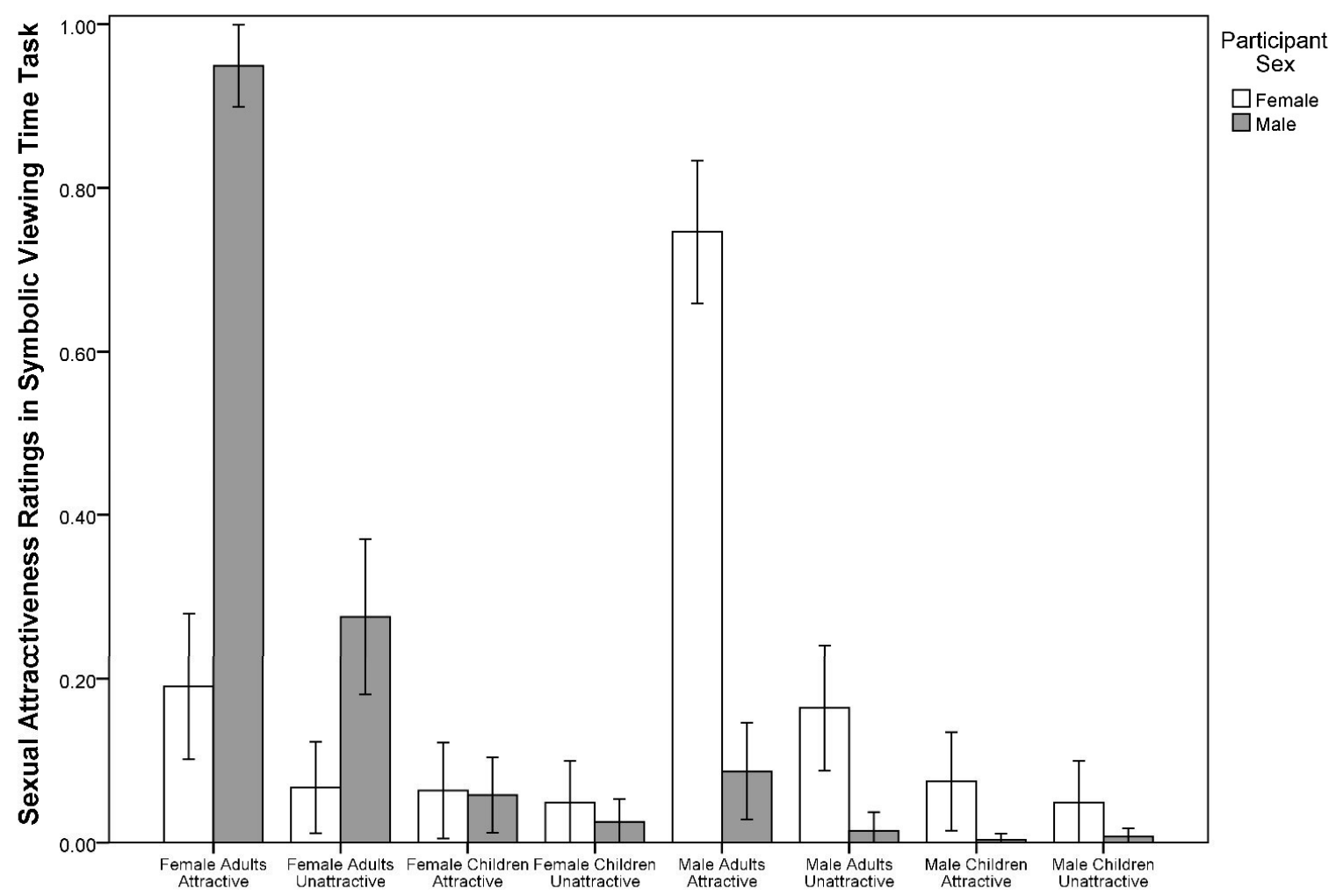


Figure 5

Response latencies ( $\pm C_{95 \%}$ ) as a function of Participant Sex, Stimulus Sex, and Stimulus Sexual Maturity in the symbolic viewing time task.

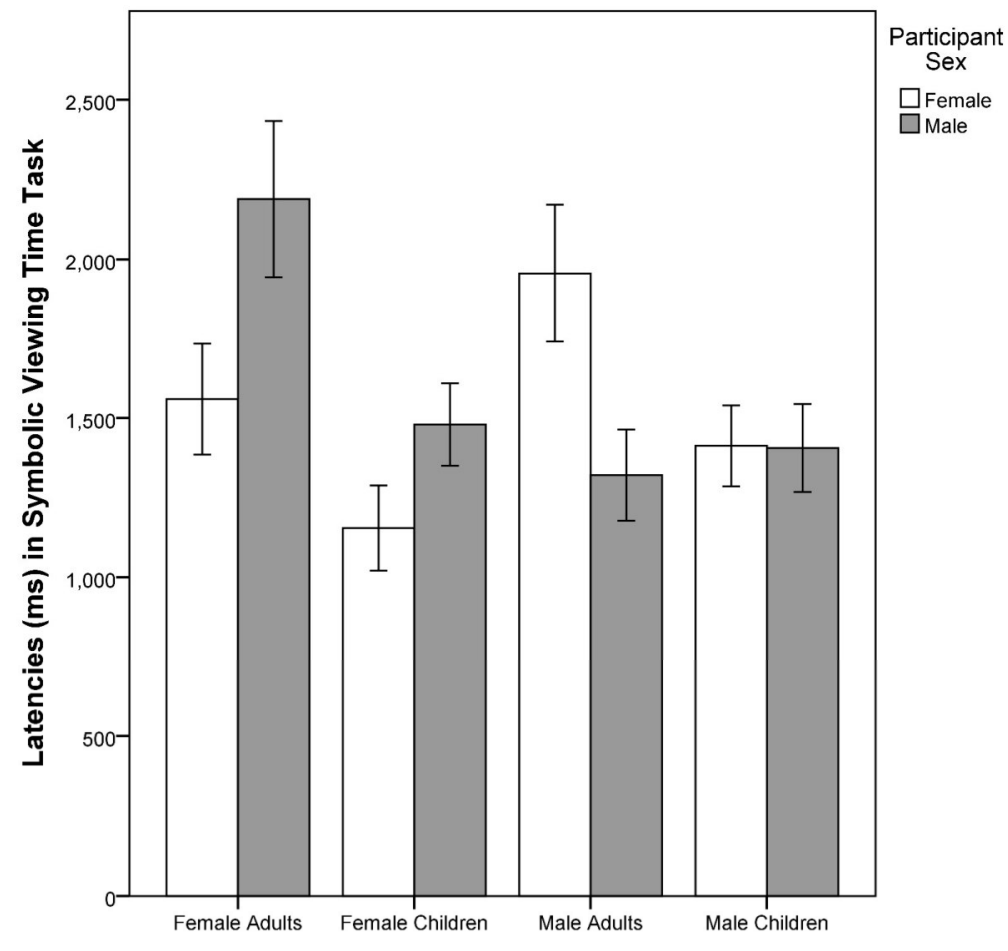




\section{Electronic Supplement}

\section{Figure A}

Response latencies $\left( \pm C I_{95 \%}\right)$ as a function of Participant Sex, Stimulus Sex, Stimulus Sexual Maturity, and Stimulus Attractiveness in the symbolic viewing time task.

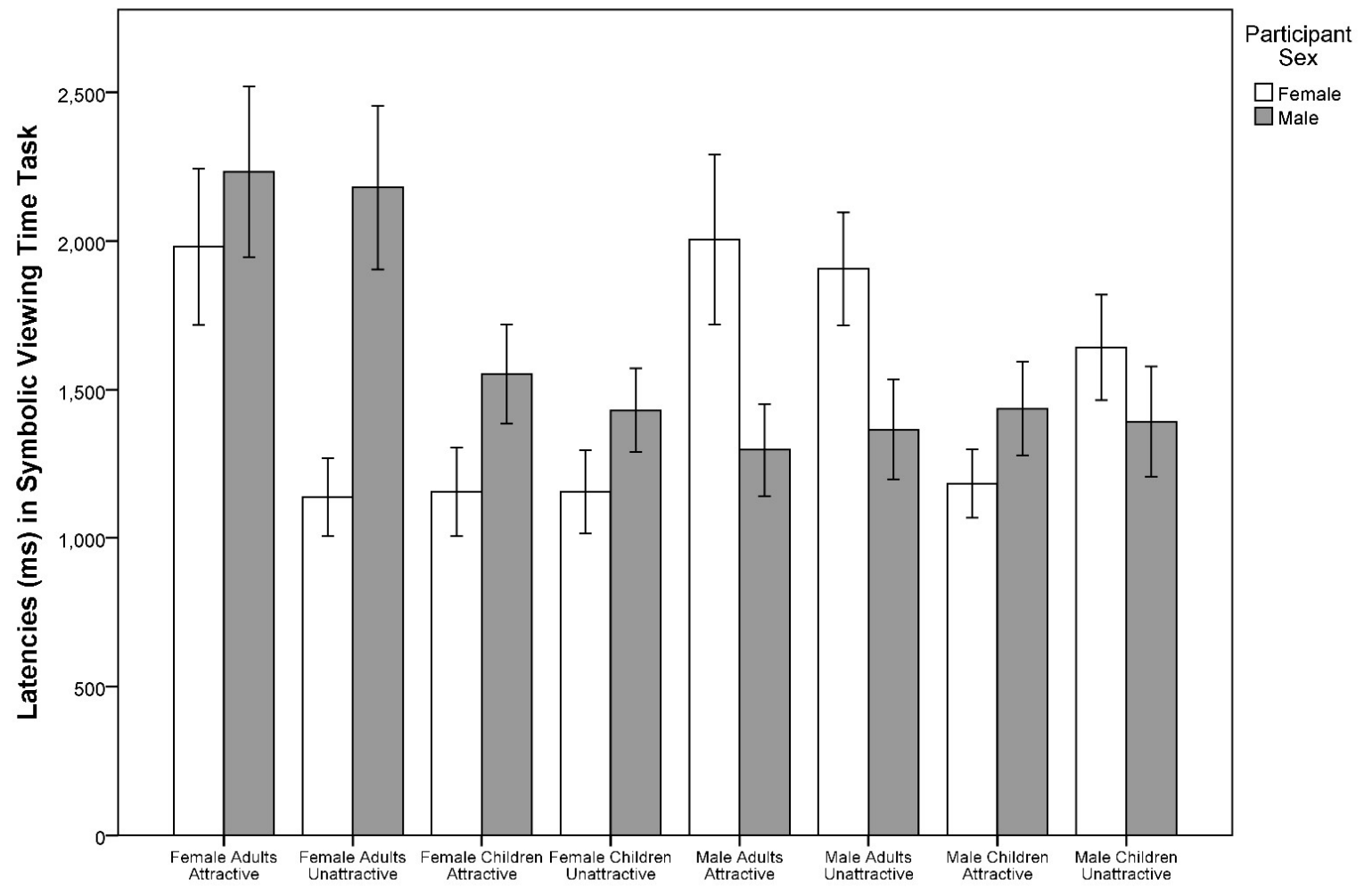

